\title{
On the relation between visual surround and motion aftereffect velocity
}

\author{
HERBERT H. BELL \\ University of Tennessee at Nashville, Nashville, Tennessee 37203 \\ STEPHEN W. LEHMKUHLE \\ Vanderbilt University, Nashville, Tennessee 37240 \\ and \\ DAVID H. WESTENDORF \\ University of Arkansas, Fayetteville, Arkansas 72701
}

\begin{abstract}
Two experiments examined the effect of changes in the visual surround upon the velocity of motion aftereffects. Experiment I showed that introduction or reintroduction of a patterned surround midway through the test period was sufficient to produce an increase in apparent velocity. However, a greater increase was observed when a patterned surround instead of a dark homogeneous surround had been used during the induction period. Experiment II demonstrated that luminance change was also sufficient to produce an increase in apparent velocity, although the extent of the increase was not as great as that produced through the use of the patterned surround in Experiment $I$. These results indicate that a change in stimulus surround is sufficient to produce an increase in the velocity of a motion aftereffect and that the extent of the increase is dependent upon the characteristics of both the induction and test surrounds.
\end{abstract}

Strelow and Day (1971) showed that a motion aftereffect (MAE) decays more rapidly when the test stimulus is viewed within a dark homogeneous visual field than when viewed in the presence of a patterned surround. In addition, they demonstrated that the reintroduction of a patterned surround midway through the test period produced an increase in the apparent velocity of the MAE. The authors concluded that reintroduction of a patterned surround "triggered" a stored MAE by removing an unspecified inhibitory process from the neural mechanisms responsible for the MAE. Strelow and Day $(1971,1975)$ further concluded that neural mechanisms responsible for the MAE receive information about stationary as well as moving contours.

The purpose of the present investigation was to examine in greater detail the effect of a change in the visual field surrounding the test stimulus upon the apparent velocity of the MAE. Specifically, this study sought (a) to determine if the presence of a patterned surround during the induction period was a necessary condition in order for that surround to produce an increase in the apparent velocity of a MAE during the test

This investigation was supported in part by National Institutes of Health Grant EY00590 to Robert Fox and a University of Tennessee Alumni research grant to Herbert $\mathrm{H}$. Bell. We are grateful to Professor Fox for his many helpful comments on an earlier version of this manuscript. Portions of this paper were presented at the meeting of the Midwestern Psychological Association, Chicago, May 1975. Reprint requests may be sent to Herbert H. Bell, Division of Arts and Sciences, University of Tennessee at Nashville, Nashville, Tennessee 37203. period, and (b) to determine if an increase in surround luminance was sufficient to produce an increase in the apparent velocity of the MAE.

\section{EXPERIMENT I}

This experiment was designed to examine the relative importance of the patterned surround during the induction period. In the experiment reported by Strelow and Day (1971), the inducing stimulus was always surrounded by a patterned stimulus and this same patterned surround was reintroduced midway through the test period. Unfortunately, this procedure does not allow one to determine the relative importance of the patterned surround present during induction. It is possible that the presence of a patterned surround during the induction period is unnecessary for the increase in MAE velocity produced by the introduction of a patterned surround during the test period. Indeed, Day and Strelow (1971) reported that a patterned surround visible throughout the test period increased the magnitude of the MAE even when the inducing stimulus was presented within a dark homogeneous surround. This experiment sought to examine the importance of the patterned surround during the induction period by inducing the MAE either with or without a patterned surround and then initially estimating the MAE in the absence of any visible surround. Midway through the test period, the patterned surround was introduced to determine if the increase in MAE velocity reported by 
Strelow and Day required the presence of a patterned surround during induction.

\section{Method}

Apparatus and stimuli. The apparatus was fashioned from optic bench components and mounted on a steel table. Two steel rails were mounted perpendicular to one another on the table, with the surround and target stimuli positioned along each rail. These stimuli were spatially superimposed through the use of mirrors. The viewing port was fitted with a chinrest and trial frames; the right eye of these trial frames contained a $2-\mathrm{mm}$ artificial pupil, and the left eye was occluded. The entire apparatus was fitted with a lightproof cover and internal baffles to eliminate stray light.

The target stimulus used as both the induction and stationary test stimulus was a vertical sine-wave grating generated on an oscilloscope (Tektronix 535) through the use of a technique similar to that employed by Campbell and Green (1965). A high-frequency triangular signal was applied to the vertical axis of the oscilloscope, and a sinusoidal signal was connected to the $Z$ axis and the trigger input through a motor-driven synchroresolver. The synchro-resolver was used to change the phase relation between the $Z$-axis signal and the trigger input, thereby causing the grating to drift across the face of the oscilloscope at $2.7 \mathrm{~Hz}$ during the induction period. The oscilloscope was mounted on one rail of the apparatus at a viewing distance of $82 \mathrm{~cm}$, and the face of the oscilloscope was masked to form a circular aperture $2.8 \mathrm{deg}$ in diameter. The space average luminance, contrast ratio, and spatial frequency of this grating were $3.0 \mathrm{~cd} / \mathrm{m}^{2}, .34$, and $2.7 \mathrm{c} / \mathrm{deg}$, respectively. A small black fixation point was marked on the face of the oscilloscope, and the observers were instructed to maintain fixation throughout the induction and test periods.

The patterned surround was a vertically oriented squarewave grating with a space average luminance of $3.1 \mathrm{~cd} / \mathrm{m}^{2}$, a spatial frequency of $2.0 \mathrm{c} / \mathrm{deg}$, and a contrast ratio of .87 . This patterned surround subtended $9 \mathrm{deg}$ of visual angle and contained an opaque centered disk, $2.8 \mathrm{deg}$ in diameter, that optically coincided with the circular aperture through which the oscilloscope was viewed. This opaque disk insured that presentation of the surround did not alter the luminance or contrast ratio of the target stimulus. In order to approximate the spectral characteristics of the target stimulus, a green filter was positioned in front of the surround.

Procedure. Four paid volunteers $(\$ 2 / \mathrm{h})$ and one of the authors (S. W. L.) served as observers. Each observer received three presentations of each experimental condition. The order of presentations was randomly determined for each observer, and successive conditions within each session were separated by rest intervals of at least $1.5 \mathrm{~min}$.

Prior to the experimental sessions, the observers participated in a preliminary session, during which they were pretested to insure that a MAE was spontaneously reported. No observer failed to spontaneously report a MAE. Following this pretest, observers were given 12 practice trials during which they continuously estimated the velocity of a MAE by making appropriate adjustments in the position of a lever. The lever was mounted to the right of the observer and pivoted through an arc of approximately $120 \mathrm{deg}$. Observers oriented the lever vertically to signal the initial velocity of the MAE and to the far right to signal the absence of any MAE; they used intermediate positions to represent intermediate velocities. The lever was connected to a potentiometer, which varied the voltage to a strip chart recorder, so that pen displacement was directly proportional to the position of the lever. Pen displacement, in centimeters, was used to indicate the velocity of the MAE.

Three experimental conditions were employed. In each condition, the target stimulus was visible throughout the induction and test periods. Whenever the patterned surround was absent, the target stimulus was seen within a dark featureless surround with a luminance of less than $.01 \mathrm{~cd} / \mathrm{m}^{2}$. The MAE was induced by viewing the drifting target stimulus for $45 \mathrm{sec}$, and each test period lasted $30 \mathrm{sec}$. In condition PDP, the patterned surround was present during induction, absent for the first 15 sec of the test period, and reintroduced for the final $15 \mathrm{sec}$ of the test period. In condition PDD, the patterned surround was present during the $45-\mathrm{sec}$ induction period and absent for the entire test period. In condition DDP, the patterned surround was absent for both the induction period and the first $15 \mathrm{sec}$ of the test period; during the final $15 \mathrm{sec}$ of the test period, the patterned surround was visible.

\section{Results}

Since a preliminary analysis of the data indicated that estimates of MAE velocity did not change in any consistent manner across the three replications of each experimental condition, these replications were averaged prior to further data analysis. The mean estimates of MAE velocity for each experimental condition are shown in Figure 1 at $1 \mathrm{sec}$ intervals over the entire test period. During the first $15 \mathrm{sec}$ of the test period, the velocity estimates were approximately equal and declined at approximately the same rate. For condition PDD, the test stimulus appeared to be stationary for each observer prior to the end of the $30-\mathrm{sec}$ test period. This condition served as a control to insure the validity of the velocity estimation procedure and to provide an indication of the extent of the velocity increases obtained when a patterned surround was introduced.

Figure 1 indicates that the introduction of a patterned surround midway through the test period produced a dramatic increase in the apparent velocity of the MAEs for both condition PDP and condition DDP. The velocity estimates increased very rapidly for both conditions and reached their new maxima approximately $3 \mathrm{sec}$ after the introduction of the patterned surround. The magnitude of the increase was dependent upon whether or not the patterned surround was present during induction. The mean velocity estimate for condition PDP was $3.8 \mathrm{~cm}$, while the corresponding velocity estimate for condition DDP was $2.6 \mathrm{~cm}[t(4)=3.54$, $p<.05]$.

\section{EXPERIMENT II}

The first experiment clearly demonstrates that introduction of a pattemed surround during the test period increases the apparent velocity of a MAE regardless of whether or not the patterned surround had been present during induction. Experiment II was designed to determine if a similar increase in the apparent velocity of a MAE could be produced merely by a change in the luminance of a homogeneous surround. Afterimages, which fade rapidly when viewed against an unchanging background, frequently reappear following a sudden change in the luminance of the background (Barlow \& Sparrock, 1964; MacLeod \& Hayhoe, 1974; Magnussen \& Torjussen, 1974). Although afterimages and MAEs reflect different underlying visual mechanisms, at least some of the increase in the apparent velocity of the 


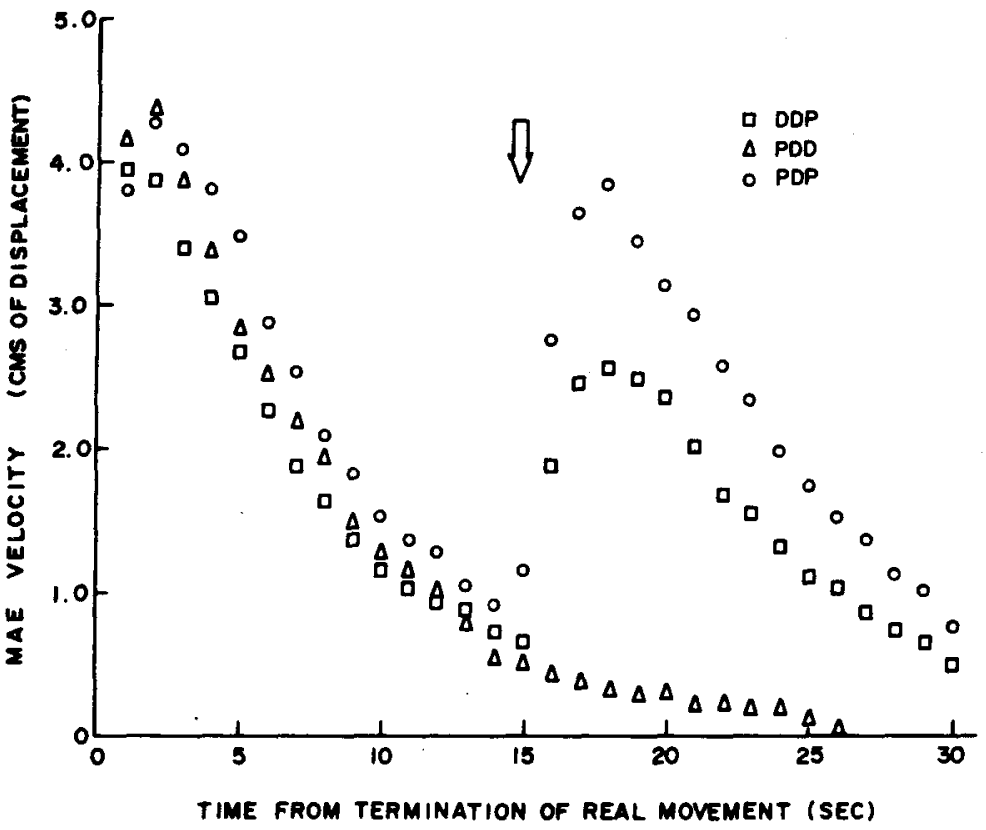

Figure 1. Mean estimated velocity of the MAE as a function of experimental condition throughout the 30-sec test period. The arrow mdicates the introduction of the patterned surround for conditions DDP and PDP.

MAE observed following the introduction of the patterned surround in the first experiment may be due to the sudden increase in the luminance of the surround produced by the onset of the patterned surround. Indeed, Holland (1965) and Spigel (1965) have suggested that changes in stimulus properties may affect inhibitory processes related to the MAE. In order to test this possibility, a homogeneous surround with a luminance equal to the space average luminance of the patterned surrounds employed in Experiment I was introduced midway through the test period.

\section{Method}

Two paid volunteers $(\$ 2 / \mathrm{h})$ from Experiment I and one of the authors (S. W. L.) served as observers in this experiment. Each observer received three presentations of each of the two experimental conditions in randomized order. In condition $\mathrm{HDH}$, a homogeneous surround with a space average luminance of $3.1 \mathrm{~cd} / \mathrm{m}^{2}$ was present during the $45-\mathrm{sec}$ induction period, absent for the first $15 \mathrm{sec}$ of the test period, and reintroduced for the final $15 \mathrm{sec}$ of the test period. In condition DDH, the surround was dark (space average luminance less than $.01 \mathrm{~cd} / \mathrm{m}^{2}$ ) and featureless for both the $45-\mathrm{sec}$ induction period and the first $15 \mathrm{sec}$ of the test period. The same homogeneous surround employed in condition HDH was introduced for the final $15 \mathrm{sec}$ of the test period in condition DDH. The space average luminances of both the homogeneous and dark surround were equivalent to the space average luminances of the patterned and dark surrounds employed in Experiment I. All other details relating to the target stimulus and procedure were identical to Experiment I.

\section{Results}

Since no systematic changes were found in the velocity estimates across the three replications of each experimental condition, these replications were averaged for each observer. Figure 2 shows that the velocities of the MAEs decreased in a manner similar to that observed in Experiment $I$ and then increased following the intro- duction of the homogeneous surround, However, unlike Experiment I, there was no differential increase in the velocity of the MAE as a result of reintroducing the homogeneous surround that had been present during induction. The maximum mean velocity estimates during the final $15 \mathrm{sec}$ of the test period were $2.0 \mathrm{~cm}$ for condition $\mathrm{HDH}$ and $2.1 \mathrm{~cm}$ for condition DDH $(p>.05)$. In addition, these maximum increases in MAE velocity were lower than the velocity increases produced by the introduction or reintroduction of the patterned surround in Experiment $I$ for each of the three observers who participated in both experiments.

\section{DISCUSSION}

This investigation demonstrates three important facts concerning the effect of a change in the visual surround upon the velocity of a MAE: First, a large increase in the luminance of the surround is sufficient to produce an increase in MAE velocity. Second, a patterned surround introduced during the test period produces an increase in MAE velocity regardless of whether or not that patterned surround was present during induction. Third, the increase in MAE velocity following the introduction of a patterned surround is greater when the patterned surround is present during induction than when the inducing stimulus is presented within a dark homogeneous field.

The results of this investigation, together with the demonstrations of Strelow and Day (1971, 1975), indicate that additional processes must be added to the basic models used to describe the neural mechanisms underlying the MAE (e.g., Barlow \& Hill, 1963; Sekuler \& Pantle, 1967; Sutherland, 1961). One important question for subsequent investigation is to determine 


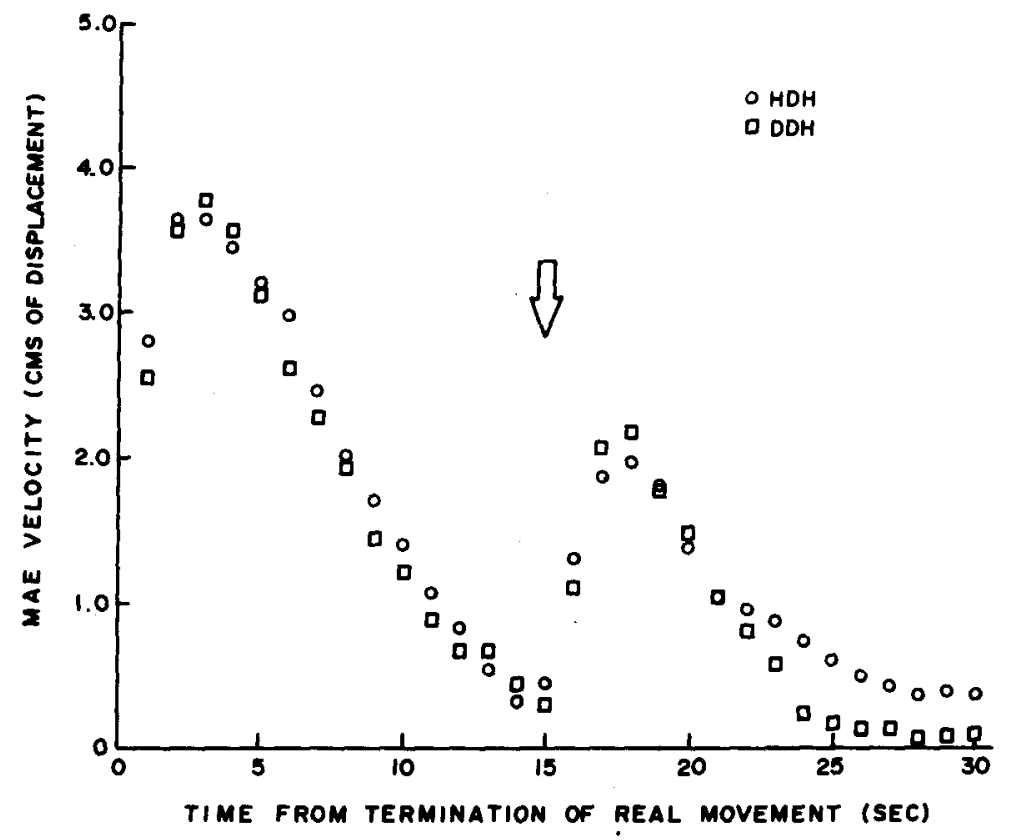

Figure 2. Mean estimated velocity of the MAE as a function of experimental condition throughout the 30-sec test period. The arrow Indicates the introduction of , the homogeneous surround.

the mechanisms through which surround changes increase the velocity of the MAE. Surround manipulations may directly affect either direction specific units of the visual system or higher visual centers that utilize the output of these direction-specific mechanisms. In addition, the greater increase in MAE velocity observed when the patterned surround was present during induction suggests a specificity of neural mechanisms that are responsive not only to movement but also to static properties of the visual field (e.g., Bonnet \& Pauthas, 1972; Favreau, Emerson, \& Corballis, 1972; Mayhew \& Anstis, 1972; Strelow \& Day, 1975). However, any attempt to examine such specificity through manipulation of the visual surround must take into account the effect of luminance change independently of any pattern manipulation.

\section{REFERENCES}

Barlow, H. B., \& Hill, R. M. Evidence for a physiological explanation of the waterfall illusion and figural aftereffects. Nature, 1963, 200, 1434-1435.

Barlow, H. B., \& SPARrock, J. M. B. The role of afterimages in dark adaptation. Science, 1964, 144, 1309-1314.

Bonnet, C., \& Pauthas, V. Interactions between spatial and kinetic dimensions in movement aftereffect. Perception \& Psychophysics, 1972, 12, 193-200.

Campeell, F. W., \& GReEN, D. G. Optical and retinal factors affecting visual resolution. Journal of Physiology, 1965 , 181, 567-593.
DAY, R. H., \& STrelow, E. R. Visual aftereffect of movement: Partial or complete reduction in the absence of a patterned surround. Nature, 1971, 230, 55-56.

Favreau, O. P., Emerson, V. F., \& Corballis, M. C. Motion perception: A color-contingent aftereffect. Science, 1972, 176, 78-79.

Holland, H. C. The spiral after-effect. Oxford: Pergamon Press, 1965.

MacLEod, D. I. A., \& Hayhoe, M. Rod origin of prolonged afterimages. Science, 1974, 185, 1171-1172.

MaYhew, J. E. W., \& Anstis, S. M. Movement aftereffects contingent on color, intensity, pattern. Perception \& Psychophysics, 1972, 12, 77-85.

Magnussen, S., \& Tonussen, T. Sustained visual afterimages. Vision Research, 1974, 14, 743-744.

Sekuler, R., \& Pantle, A. A model for aftereffects of seen motion. Vision Research, 1967, 7, 427-439.

SPIGEL, I. M. Problems in the study of visually perceived movement: An introduction. In I. M. Spigel (Ed.), Readings in the study of visually perceived movement. New York, Harper and Row, 1965.

Strelow, E. R., \& DAY, R. H. Aftereffect of visual movement: Storage in the absence of a patterned surround. Perception \& Psychophysics, 1971, 9, 485-486.

STRELOW, E. R., \& DAY, R. H. Visual movement aftereffect: Evidence for independent adaptation to moving target and stationary surround. Vision Research, 1975, 15, 117-121.

Sutherland, N. S. Figural aftereffects and apparent size. Quarterly Journal of Experimental Psychology, 1961, 13. 222-228.

(Received for publication December 22. 1975; revision accepted February 9. 1976.) 